Original Paper http://ajol.info/index.php/ijbcs http://indexmedicus.afro.who.int

\title{
Détermination de la teneur en résidus de cinq pesticides organochlorés dans le lait des grands bassins laitiers au Mali-Sud
}

\author{
Boubacar Madio dit Aladiogo MAÏGA ${ }^{1 *}$, Ibrahima KONÉ ${ }^{2}$, Oumou HAÏDARA ${ }^{1}$, \\ Salimata KONÉ ${ }^{3}$, Fousseni DIALLO ${ }^{1}$, Alimata BERTHÉ ${ }^{1}$, Idrissa TRAORÉ ${ }^{1}$ et \\ Aminata SISSOKO ${ }^{1}$
}

\author{
${ }^{1}$ Laboratoire Central Vétérinaire, BP 2295, Tél : (+223) 20243344 Km8, Sotuba, Bamako-Mali. \\ ${ }^{2}$ Institut de Formation Professionnelle, BP 53, Tél : (+223) 21723852 Konobougou-Mali. \\ ${ }^{3}$ Agence Nationale de la Sécurité Sanitaire des Aliments, BPE : 2362 Tél : (+223) 202207 54, Bamako-Mali. \\ *Auteur correspondant ; E-mail : aladiogo2@gmail.com
}

\section{REMERCIEMENTS}

Nous remercions le Laboratoire Central Vétérinaire (LCV) du Ministère de l'Innovation et de la Recherche Scientifique du Mali pour avoir financé à travers le "Fonds Etude et Recherche » les travaux de cette étude.

\section{RESUME}

Les pesticides organochlorés à cause de leur stabilité chimique persistent et s'accumulent dans différents milieux des régions où ils sont utilisés régulièrement et pénètrent dans la chaîne alimentaire. Leur grande liposolubilité fait qu'ils peuvent être retrouvés dans le lait. La présente étude, réalisée au niveau des zones des grands bassins laitiers au Mali-Sud, vise à déterminer les teneurs en résidus de cinq pesticides organochlorés dans le lait de vache. Les échantillons de lait collecté $(\mathrm{n}=100)$ ont été prélevés au niveau de cinq sites des régions de Koulikoro et Sikasso. Ils ont été extraits et analysés à l'aide d'un chromatographe en phase gazeuse Agilent 7890A équipé d'un détecteur à captures d'électrons (ECD). L'analyse des résultats a montré une contamination du lait par les cinq pesticides organochlorés. La charge moyenne totalisée par type de pesticide a été de : $0,97 \pm 0,45 \mathrm{mg} / \mathrm{L}$ pour le dichloro-diphenyl-trichloroéthane (DDT) détecté dans $24 \%$ des échantillons de lait, $0,61 \pm 0,53 \mathrm{mg} / \mathrm{L}$ pour l'endosulfane $\beta(18 \%), 0,53 \pm 0,44 \mathrm{mg} / \mathrm{L}$ pour l'endosulfane $\alpha$ (19\%), 0,41 $\pm 0,29 \mathrm{mg} / \mathrm{L}$ pour $13 \%$ de dieldrine et $0,08 \pm 0,10 \mathrm{mg} / \mathrm{L}$ pour seulement $4 \%$ de lindane. Ces teneurs expliquent l'influence de l'utilisation des pesticides sur la bioconcentration des cinq pesticides dans le lait de vache des zones de production cotonnière.

(C) 2018 International Formulae Group. All rights reserved.

Mots clés : Bioconcentration, contamination, charge moyenne, production cotonnière, lait de vache et Mali.

\section{Determination of the residues content of five organochlorine pesticides in the milk of large milk basins in southern Mali}

\begin{abstract}
Organochlorine pesticides because of their chemical stability persist and accumulate in different environments in areas where they are used regularly and enter the food chain. Their great liposolubility means that they can be found in milk. This study, conducted at the level of the large dairy catchment areas in southern
\end{abstract}


Mali, aims to determine the residue levels of five organochlorine pesticides in local milk. 100 samples of raw milk were collected from five sites in the Koulikoro and Sikasso regions. They were extracted and analyzed using an Agilent 7890A gas chromatograph equipped with an electron capture detector (ECD). The analysis of the results showed a contamination of the milk by five organochlorine pesticides. The mean totalized load per type of pesticide was: $0.97 \pm 0.45 \mathrm{mg} / \mathrm{L}$ for dichlorodiphenyltrichloroethane (DDT) detected in $24 \%$ of the 100 milk samples, $0.61 \pm 0.53 \mathrm{mg} / \mathrm{L}$ for endosulfan $\beta(18 \%), 0.53 \pm 0.44 \mathrm{mg} / \mathrm{L}$ for endosulfan $\alpha(19 \%), 0.41 \pm 0.29$ $\mathrm{mg} / \mathrm{L}$ for $13 \%$ of dieldrin and $0.08 \pm 0,10 \mathrm{mg} / \mathrm{L}$ for only $4 \%$ of lindane. These levels indicate a bioconcentration of these pesticides in the milk of cows that ingested surface water and / or herbs and crop products from treated areas, or even inhalation of air polluted by these compounds.

(C) 2018 International Formulae Group. All rights reserved.

Keywords: Bioconcentration, contamination, average load, cotton production, cattle milk and Mali.

\section{INTRODUCTION}

Le Mali est un vaste pays à vocation agropastorale, situé au cœur de l'Afrique occidentale. Son agriculture connaît depuis l'avènement de la Compagnie Malienne de Développement des Textiles (CMDT), une large utilisation d'intrants chimiques dont un volume total de 2794992 doses d'insecticides, 854100 litres d'herbicides et 298500 sachets de fongicides, a été utilisé comme pesticides pour la production du coton en 2017 (CMDT, 2018). Son agriculture est fortement tributaire du sous-secteur de l'élevage de par l'essor de la culture attelée. Les revenus obtenus de la vente des produits agricoles (coton, céréales, tubercules) contribuent à l'acquisition du cheptel des agro éleveurs. Mais de plus en plus, les éleveurs et agro éleveurs tirent leurs revenus de la vente du lait et des produits laitiers. De ce fait, certains ont inscrit leur exploitation dans une dynamique de chaîne de valeurs à travers les mini-laiteries. Les revenus de la vente du lait et des produits laitiers contribuent pour $18 \%$ au revenu annuel des producteurs qui sont affiliés à une laiterie dans les zones périurbaines (DNPIA, 2012). Cependant, cette filière est confrontée à une série de potentiels contaminants du lait comme les résidus d'antibiotiques et de pesticides (Bonfoh et al., 2003).

En Afrique de l'Ouest, seuls les agents microbiens pathogènes, les résidus de pesticides et les aflatoxines ont fait l'objet de travaux ou d'études dans le cadre d'une démarche de protection dans le domaine de la sécurité sanitaire des aliments destinés à la consommation humaine. Ces dangers ont été les plus relatés comme menaçant la santé publique (Mensah et al., 2014). La contamination du lait et des produits laitiers par les résidus de pesticides constitue un risque majeur pour les consommateurs (David, 2004) mais aussi une question préoccupante, tant pour des raisons économiques que sanitaires (Grillet et al., 2005).

Des travaux conduits dans la zone de production cotonnière de la région de Sikasso ont permis de signaler la contamination des sols et des eaux de puits (Dem et al., 2007). Une autre étude réalisée dans la même zone a également révélé la présence de résidus de pesticides dans les produits maraichers (Amadou et al., 2014). Sur la base des résultats du suivi des polluants organiques persistants qui ont révélé la présence du DDT dans le lait maternel ainsi que de la dieldrine et du lindane dans l'air ambiant au Mali (UNEP, 2012) et de l'inventaire de 1100 tonnes de pesticides obsolètes dans le pays (PASP, 2009), une liste prioritaire de cinq pesticides organochlorés (dieldrine, DDT, endosulfane $\alpha$, endosulfane $\beta$ et lindane) à rechercher a été définie. Une étude récente réalisée au Cameroun fait clairement ressortir que les pesticides organochlorés sont assez bien utilisés malgré leur interdiction (Tchamadeu et al., 2017). L'objectif général de l'étude a été d'évaluer la teneur en résidus de pesticides organochlorés dans le lait de vache produit dans des sites des bassins laitiers des régions de Koulikoro et de Sikasso. 


\section{MATERIEL ET METHODES \\ Zone et période d'étude}

L'étude de prospection s'est déroulée en 2013 et les prélèvements ont été effectués en deux saisons en mai (S1 : saison sèche) et en septembre (S2: saison pluvieuse). Les sites dotés chacun d'une mini-laiterie ont été choisis en ciblant deux zones distinctes : la zone 1 de grande production cotonnière (Koumantou, Ouéléssebougou et Sido) et la zone 2 de faible production cotonnière (Tienfala et Kasséla). Ils sont localisés au bord des axes routiers reliant les capitales régionales (Koulikoro, Ségou et Sikasso) à la ville de Bamako (figure 1). Ils se trouvent dans la zone soudanienne du Mali caractérisée par une pluviométrie annuelle comprise entre 800 et $1100 \mathrm{~mm}$ avec une production moyenne annuelle de lait de vache estimée à environ 79437 tonnes pour la région de Koulikoro contre 88242 tonnes pour la région de Sikasso (DNPIA, 2016).

\section{Matériel biologique}

Le matériel biologique de cette étude est constitué d'échantillons de lait frais de vaches

\section{Echantillonnage}

Des prélèvements d'échantillons de lait ont été effectués au niveau de cinq sites organisés en mini-laiteries, représentant les principaux bassins laitiers des régions de Koulikoro et Sikasso. Pour chacun des sites, quatre points de prélèvement (ferme, centre de collecte, revendeurs et mini-laiterie) ont été retenus et cinq échantillons composites de lait frais (fermes (1), centres de collecte (1), revendeurs (1) et (2) pour la mini-laiterie) d'un volume total de $500 \mathrm{~mL}$ chacun ont été prélevés $(5$ sites $\times 4$ points de prélèvement $\times 5$ échantillons par site). Les échantillons ( $\mathrm{n}=100)$ tous prélevés les matins maximum 1 heure de temps après la traite des vaches, dans des flacons en verre, ont été étiquetés et placés sous plaques réfrigérantes. Ils sont ensuite transportés au laboratoire plus tard 2 heures après les prélèvements où ils ont été conservés au réfrigérateur avant analyse.
Traitement et analyse des échantillons (ISO 3890-1, 3890-2 : 2009)

Les échantillons ont été traités et analysés selon les méthodes d'analyses validées par le Laboratoire Central Vétérinaire suivant les normes (ISO 3890-1 : 2009) et (ISO 3890-2 : 2009) pour le dosage des résidus de pesticides organochlorés dans le lait.

\section{Extraction, concentration et purification}

L'étape d'extraction a pour objectif d'extraire les pesticides des échantillons de lait. Une prise d'essai de $10 \mathrm{~mL}$ de lait a été prélevée de chaque échantillon et malaxée avec $60 \mathrm{~g}$ de sulfate de sodium anhydre $\left(\mathrm{Na}_{2} \mathrm{SO}_{4}\right)$ jusqu'à l'obtention d'un mélange homogène. Ce dernier est transvasé dans une colonne chromatographique contenant de la laine de verre et du sulfate de sodium anhydre. Cet ensemble est élué avec $240 \mathrm{~mL}$ de dichlorométhane. L'éluat est recueilli dans un ballon de $500 \mathrm{~mL}$ et préconcentré à $35^{\circ} \mathrm{C}$ à l'évaporateur rotatif jusqu'à environ $50 \mathrm{~mL}$. Cette portion est transvasée dans un ballon de $100 \mathrm{~mL}$ puis préconcentrée jusqu'à sec cette fois. Le résidu est récupéré dans le ballon tout de suite avec $50 \mathrm{~mL}$ d'hexane puis purifié à l'aide du Florisil et un mélange (4/5 d'hexane et $1 / 5$ de dichloromethane). Le produit obtenu est préconcentré jusqu'à $10 \mathrm{~mL}$. Ce volume final est conditionné dans de l'hexane et transvasé dans une fiole de 10 $\mathrm{mL}$. Enfin, un prélèvement de $1,5 \mathrm{ml} \mathrm{du}$ volume final est effectué et transvasé dans une vial pour l'analyse au chromatographe en phase gazeuse (CPG).

\section{Solutions de standards analytiques}

Les standards analytiques individuels de référence des cinq pesticides de pureté varient entre 96,3 et $98,5 \%$ et ont été fournis par le fabricant (Ehrenstorfer $\mathrm{GmbH}$ Reference Augsburg-Germany). Une solution individuelle de chaque étalon nécessaire pour l'identification et la quantification a été préparée par dilution de 
la solution mère dans l'hexane «pour résidus ». Mais la solution du mélange des cinq standards a été préparée par dilution de petites quantités dans de l'hexane à $5 \mathrm{mg} / \mathrm{L}$. Puis une gamme d'analyse de cinq niveaux différents à plusieurs concentrations $(0,0125 ; 0,25 ; 0,5 ; 0,1$ et 0,125 $\mu \mathrm{g} / \mathrm{mL}$ ) a été préparée et utilisée pour l'étalonnage du CPG.

\section{Analyse instrumentale}

Les extraits des échantillons ont été analysés au chromatographe en phase gazeuse (Agilent 7890A) muni d'un détecteur à capture d'électrons (ECD) et d'un logiciel d'exploitation CHEMSTATION. Une colonne capillaire RTX5 Crossbond, 30 mètres $\mathrm{x} 250 \mu \mathrm{m}$ de diamètre interne x $0.50 \mu \mathrm{m}$ d'épaisseur de film, phase stationnaire $5 \%$ diphenyl-95\% dimethyl polysiloxane a été utilisée. Une colonne de type HP-5 (5\% Phenyl methyl Siloxane) a permis de confirmer le statut des échantillons déjà analysés (négatifs et positifs). L'injecteur était en mode sans division (splitless) à une température de $280{ }^{\circ} \mathrm{C}$ avec l'azote comme gaz vecteur et d'appoint de $60 \mathrm{ml} / \mathrm{mn}$ et un débit constant de 1,6 $\mathrm{mL} / \mathrm{mn}$. La programmation de la température du four en trois paliers a été la suivante: un palier initial compris entre 70 $150{ }^{\circ} \mathrm{C}$ suivi d'un palier secondaire de 150 $200{ }^{\circ} \mathrm{C}$ et un dernier de $200-280{ }^{\circ} \mathrm{C}$. Le détecteur était programmé à une température de $300{ }^{\circ} \mathrm{C}$ pour un volume de l'échantillon injecté de $1 \mu \mathrm{L}$.

\section{Taux de récupération}

Des quantités connues de solution étalon des cinq pesticides recherchés ont été ajoutées individuellement à des échantillons de lait préalablement vérifiés ne contenant pas des résidus de pesticides. La quantité récupérée a été exprimée en pourcentage de la quantité ajoutée. Pour cinq passages de chaque pesticide dopé, les taux de récupération étaient de l'ordre de $94 \%$ pour le DDT, $92 \%$ pour le lindane, $91 \%$ l'endosulfane et $90 \%$ pour la dieldrine.

\section{Traitement des données statistiques}

Après la phase analytique, les résultats bruts ont été saisis et traités sur un tableur Excel sous Windows 2010 de Microsoft puis au logiciel R version 2.13.0. Le logiciel Win Episcope version 2.0 a permis de fixer la taille d'échantillon pour une prévalence estimée à $7,8 \%$, un niveau de confiance de $95 \%$ et une marge d'erreur de $5 \%$.

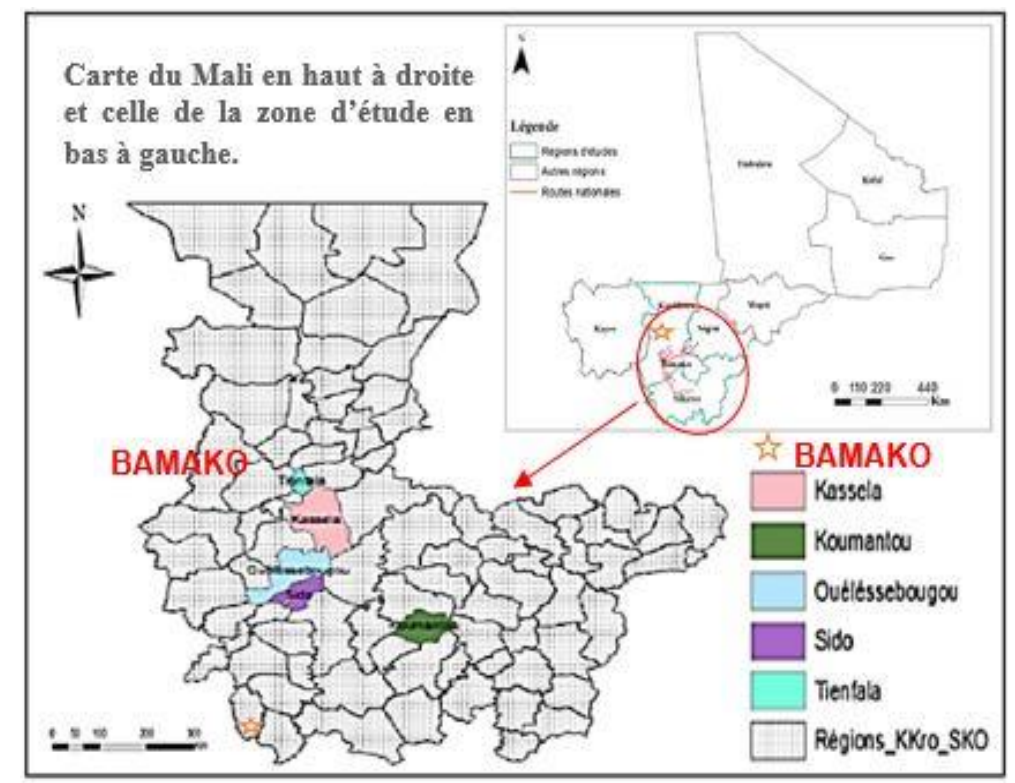

Figure 1: Localisation des sites de prélèvement. 


\section{RESULTATS}

Le nombre d'échantillons de lait contaminés par les résidus des différents pesticides en fonction des sites de production est présenté dans le Tableau 1. Les teneurs exprimées en milligramme de pesticide par litre de lait $(\mathrm{mg} / \mathrm{L})$ sont consignées dans le (Tableau 2). Les situations des échantillons contaminés ou non contaminés par zone de production cotonnière et par saison de prélèvement sont consignées respectivement dans les Tableaux 3 et 4 . Et enfin les teneurs moyennes ainsi que les pourcentages de détection des pesticides quantifiés dans les échantillons de lait, sont présentées dans le Tableau 5.

\section{Situation des échantillons par zone de production cotonnière}

Les résidus des 5 pesticides (dieldrine, DDT, endosulfane $\alpha$, endosulfane $\beta$ et lindane) ont été retrouvés dans la plupart des échantillons de lait des deux zones à l'exception du lindane qui n'a pas été quantifié dans les échantillons provenant de la zone de faible production cotonnière des sites de Tienfala et Kasséla. Un total de 55 échantillons de lait prélevés dans la zone à forte production cotonnière (zone 1) étaient contaminés par les résidus des cinq pesticides recherchés contre 23 de la zone à faible production (zone 2) avec quatre pesticides quantifiés. Avec le test de Chi-II, une différence hautement significative a pu être notée $(p<0,005)$ en fonction de la zone de production cotonnière.

\section{Situation des échantillons par saison de prélèvement des échantillons}

Aussi, les résidus de ces pesticides ont été dosés dans 36 échantillons de lait en mai (S1: saison sèche) contre 42 en septembre (S2: saison pluvieuse). Cette contamination des échantillons de lait par les résidus de pesticides organochlorés augmente plus on va vers les sites de forte production cotonnière (zone 1) quelle que soit la saison de l'année (Figure 2). Mais aucune différence significative n'a été constatée ( $p=0,1475)$ selon la saison de l'année (Tableau 3). La comparaison statistique (test de Student) de ces moyennes saisonnières a montré une différence très significative $(\mathrm{p}<0,05)$ pour le DDT et l'endosulfane $\beta$, qui sont les plus utilisés en saison pluvieuse.

Concentrations des résidus de pesticides dans le lait de vache

Au total, sur les 100 échantillons de laits analysés, 78 échantillons se sont révélés contaminés par les 5 pesticides. Le Tableau 5 nous indique que la majorité des échantillons sont contaminés à des seuils différents selon les types de pesticides. Leur cumul a été respectivement de $4,86 \mathrm{mg} / \mathrm{L}$ de lait pour le DDT ; 3,07 pour l'endosulfane $\beta ; 2,66$ pour l'endosulfane $\alpha ; 2,07$ pour la dieldrine et 0,42 $\mathrm{mg} / \mathrm{L}$ pour le lindane. Ce dernier n'a pas été quantifié au niveau de la zone 2 (Kasséla et Tienfala). Les moyennes des teneurs de résidus de 5 pesticides détectés dans le lait de

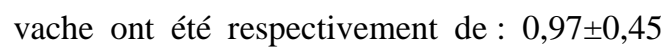
$\mathrm{mg} / \mathrm{L}$ pour le DDT détecté dans $24 \%$ des 100 échantillons de lait, $0,61 \pm 0,53 \mathrm{mg} / \mathrm{L}$ pour l'endosulfane $\beta$ (18\%), 0,53 $\pm 0,44 \mathrm{mg} / \mathrm{L}$ pour l'endosulfane $\alpha(19 \%), 00,41 \pm 0,29 \mathrm{mg} / \mathrm{L}$ pour $13 \%$ de dieldrine et $0,08 \pm 0,10 \mathrm{mg} / \mathrm{L}$ pour seulement $4 \%$ de lindane (Tableau 5). La comparaison statistique (test de Student) de ces moyennes saisonnières a montré une différence très significative $(\mathrm{p}<0,05)$ pour le DDT et l'endosulfane $\beta$, qui sont les plus utilisés en saison pluvieuse. 


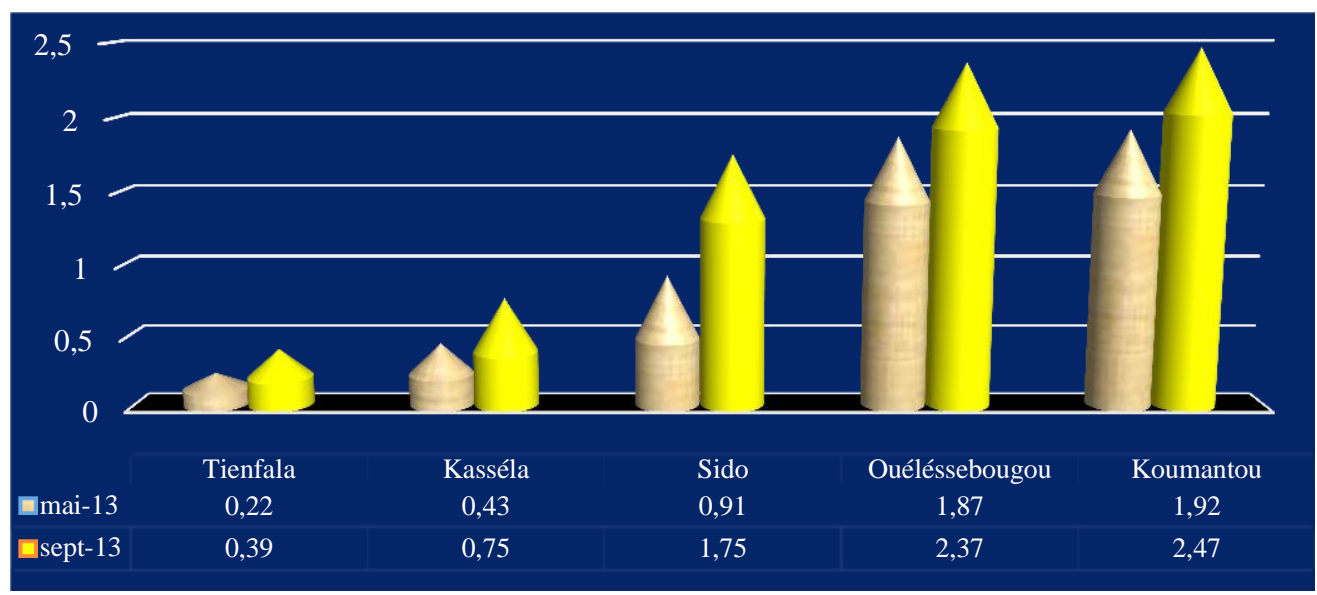

Figure 2 : Concentrations des résidus totalisés des 5 pesticides dans le lait de vache par saison de l'année (mg/L).

Tableau 1: Nombre d'échantillons contaminés par les résidus des différents pesticides détectés en fonction des sites de production.

\begin{tabular}{|c|c|c|c|c|c|c|c|c|c|c|c|c|c|c|c|c|}
\hline \multirow{2}{*}{ Sites } & \multicolumn{3}{|c|}{ Nombre Echantillons } & \multicolumn{2}{|c|}{ DDT } & \multicolumn{2}{|c|}{ Dieldrine } & \multicolumn{2}{|c|}{$\begin{array}{c}\text { Endosulfane } \\
\alpha\end{array}$} & \multicolumn{2}{|c|}{ Endosulfane $\beta$} & \multicolumn{2}{|c|}{ Lindane } & \multicolumn{3}{|c|}{ Totaux par site } \\
\hline & mai-13 & sept-13 & cumul & mai & sept & mai & sept & mai & sept & mai & sept & mai & sept & mai-13 & sept-13 & cumul \\
\hline Koumantou $^{1}$ & 10 & 10 & 20 & 3 & 3 & 1 & 2 & 2 & 2 & 2 & 2 & 1 & 1 & 9 & 10 & 19 \\
\hline Ouéléssebougou $^{1}$ & 10 & 10 & 20 & 2 & 2 & 2 & 2 & 2 & 3 & 2 & 3 & 1 & 0 & 9 & 10 & 19 \\
\hline $\operatorname{Sido}^{1}$ & 10 & 10 & 20 & 3 & 3 & 1 & 2 & 2 & 2 & 1 & 2 & 0 & 1 & 7 & 10 & 17 \\
\hline Kasséla ${ }^{2}$ & 10 & 10 & 20 & 2 & 2 & 1 & 1 & 2 & 2 & 2 & 2 & 0 & 0 & 7 & 7 & 14 \\
\hline Tienfala $^{2}$ & 10 & 10 & 20 & 2 & 2 & 0 & 1 & 1 & 1 & 1 & 1 & 0 & 0 & 4 & 5 & 9 \\
\hline Total & 50 & 50 & 100 & 12 & 12 & 5 & 8 & 9 & 10 & 8 & 10 & 2 & 2 & 36 & 42 & 78 \\
\hline
\end{tabular}

${ }^{1}$ : zone de grande production cotonnière ${ }^{2}$ : zone de faible production cotonnière mai13: saison sèche sept13: saison pluvieuse.

Tableau 2 : Concentrations moyennes en résidus de pesticides par site et par saison (mg/L).

\begin{tabular}{|c|c|c|c|c|c|c|c|c|c|c|c|c|c|c|c|c|}
\hline \multirow[t]{2}{*}{ Sites } & \multicolumn{3}{|c|}{$\begin{array}{c}\text { Nombre } \\
\text { Echantillons }\end{array}$} & \multicolumn{2}{|c|}{ DDT } & \multicolumn{2}{|c|}{ Dieldrine } & \multicolumn{2}{|c|}{$\begin{array}{c}\text { Endosulfane } \\
\alpha\end{array}$} & \multicolumn{2}{|c|}{ Endosulfane $\beta$} & \multicolumn{2}{|c|}{ Lindane } & \multicolumn{3}{|c|}{ Totaux par site } \\
\hline & mai-13 & sept-13 & cumul & mai & sept & mai & sept & mai & sept & mai & sept & mai & sept & mai-13 & sept-13 & cumul \\
\hline Koumantou $^{1}$ & 10 & 10 & 20 & 0,63 & 0,80 & 0,25 & 0,35 & 0,42 & 0,58 & 0,56 & 0,65 & 0,06 & 0,09 & 1,92 & 2,47 & 4,39 \\
\hline Ouéléssebougou ${ }^{1}$ & 10 & 10 & 20 & 0,58 & 0,70 & 0,19 & 0,40 & 0,48 & 0,53 & 0,51 & 0,62 & 0,11 & 0,12 & 1,87 & 2,37 & 4,24 \\
\hline $\operatorname{Sido}^{1}$ & 10 & 10 & 20 & 0,50 & 0,65 & 0,14 & 0,50 & 0,15 & 0,25 & 0,12 & 0,31 & $\mathrm{nq}$ & 0,04 & 0,91 & 1,75 & 2,66 \\
\hline Kasséla $^{2}$ & 10 & 10 & 20 & 0,20 & 0,40 & 0,08 & 0,10 & 0,07 & 0,10 & 0,08 & 0,15 & $\mathrm{nq}$ & $\mathrm{nq}$ & 0,43 & 0,75 & 1,18 \\
\hline Tienfala $^{2}$ & 10 & 10 & 20 & 0,15 & 0,25 & 0,02 & 0,04 & 0,03 & 0,05 & 0,02 & 0,05 & $\mathrm{nq}$ & $\mathrm{nq}$ & 0,22 & 0,39 & 0,61 \\
\hline Totaux & 50 & 50 & 100 & 2,06 & 2,80 & 0,68 & 1,39 & 1,15 & 1,51 & 1,29 & 1,78 & 0,17 & 0,25 & 5,35 & 7,73 & 13,08 \\
\hline
\end{tabular}

${ }^{1}$ : zone de grande production cotonnière ${ }^{2}$ : zone de faible production cotonnière mai13: saison sèche sept13: saison pluvieuse nq : non quantifié 
Tableau 3: Situation des échantillons de lait après les analyses par zone de production cotonnière.

\begin{tabular}{lccc}
\hline \multirow{2}{*}{ Zone } & \multicolumn{2}{c}{ Résidus de pesticides } & \multirow{2}{*}{ Total } \\
\cline { 2 - 3 } & Présents & Absents & \\
\hline Zone 1 & 55 & 5 & 60 \\
Zone 2 & 23 & 17 & 40 \\
Total & 78 & 22 & 100 \\
$p$-value & \multicolumn{2}{c}{$<005$} \\
\hline Zone 1 : zone de grande production cotonnière Zone 2 : zone de faible production cotonnière
\end{tabular}

Tableau 4: Situation des échantillons de lait de vache après les analyses par saison de l'année.

\begin{tabular}{|c|c|c|c|}
\hline \multirow{2}{*}{ Saison } & \multicolumn{2}{|c|}{ Résidus de pesticides } & \multirow{2}{*}{ Total } \\
\hline & Présents & Absents & \\
\hline mai-13 (S1) & 36 & 14 & 50 \\
\hline sept-13 (S2) & 42 & 8 & 50 \\
\hline Total & 78 & 22 & 100 \\
\hline$p$-value & \multicolumn{2}{|c|}{0,1475} & \\
\hline
\end{tabular}

Tableau 5 : Concentrations moyennes et fréquence ("\%) des 5 pesticides dans le lait de vache de mai et septembre $2013(\mathrm{mg} / \mathrm{L})$.

\begin{tabular}{|c|c|c|c|c|c|c|c|c|c|c|}
\hline & \multicolumn{2}{|c|}{ DDT } & \multicolumn{2}{|c|}{ Dieldrine } & \multicolumn{2}{|c|}{ Endosulfane $\alpha$} & \multicolumn{2}{|c|}{ Endosulfane $\beta$} & \multicolumn{2}{|c|}{ Lindane } \\
\hline & mai & sept & mai & sept & mai & sept & mai & sept & mai & sept \\
\hline Minimum & 0,15 & 0,25 & 0,02 & 0,04 & 0,04 & 0,03 & 0,02 & 0,05 & $\mathrm{nq}$ & $\mathrm{nq}$ \\
\hline $\begin{array}{l}\text { Moyenne } \\
\pm \text { Ecart type }\end{array}$ & $0,410,22$ & $0,560,23$ & $0,130,09$ & $0,280,20$ & $\begin{array}{l}0,23 \\
0,21\end{array}$ & $0,300,24$ & $\begin{array}{l}0,25 \\
0,26\end{array}$ & $\begin{array}{l}0,36 \\
0,27\end{array}$ & $\begin{array}{l}0,03 \\
0,05\end{array}$ & $0,050,05$ \\
\hline Maximum & 0,63 & 0,80 & 0,25 & 0,50 & 0,48 & 0,58 & 0,56 & 0,65 & 0,11 & 0,12 \\
\hline$\%$ de lait/POC & \multicolumn{2}{|c|}{24} & \multicolumn{2}{|c|}{13} & \multicolumn{2}{|c|}{19} & \multicolumn{2}{|c|}{18} & \multicolumn{2}{|c|}{4} \\
\hline$p$-value & \multicolumn{2}{|c|}{0,001123} & \multicolumn{2}{|c|}{0,09314} & \multicolumn{2}{|c|}{0,05016} & \multicolumn{2}{|c|}{0,02095} & \multicolumn{2}{|c|}{0,1202} \\
\hline
\end{tabular}

\section{DISCUSSION}

Les analyses ont permis la quantification de résidus des 5 pesticides organochlorés dans les échantillons de lait de vaches produit dans les zones de production cotonnière des régions de Koulikoro et Sikasso au Sud-Mali. Un des objectifs de cette étude est de vérifier l'influence de l'importance de la production cotonnière ainsi que celle de la saison de prélèvements sur la contamination des échantillons de lait. Ainsi au regard des résultats, les échantillons de la zone de grande production cotonnière (zone 1) était plus contaminés que ceux de la zone de faible production cotonnière (zone 2). Les sites de cette zone reçoivent effectivement plus de traitements réguliers des cotonniers et autres cultures vivrières avec des produits phytosanitaires pour lutter contre les insectes ravageurs des cultures que ceux de la zone 2 . Les auteurs Adjagodo et al. (2016) rapportent que les insecticides organochlorés dits « de première génération » comme l'aldrine, l'endrine, le DDT, le dieldrine, l'heptachlore et le lindane ont été massivement utilisés dans la lutte chimique contre les ravageurs du caféier, du cacaoyer et du cotonnier. $\mathrm{La}$ différence de contamination des deux zones par les pesticides organochlorés peut s'expliquer par le niveau d'utilisation des 
pesticides lié à l'intensité ou l'importance de la production cotonnière de la zone, qui est à l'origine de la contamination des sols, des eaux et des pâturages. Alors que selon Savadogo et al. (2006), la concentration des pesticides dans les sols de la zone cotonnière en l'occurrence l'endosulfane varie selon les saisons et d'après TECA-FAO (2016), la saison pluvieuse de l'année est favorable au développement des nuisibles ce qui justifie l'intensité de la lutte chimique. Cependant la saison de prélèvement n'a présenté aucune influence sur la contamination des échantillons de lait par les pesticides organochlorés. Ce qui s'explique par le fait qu'une fois utilisés les pesticides organochlorés (DDT, dieldrine, lindane et l'endosulfane) au cours d'une saison de l'année, persistent et conservent la même chance d'être retrouvés au cours de la saison sèche de la même année parce que les animaux sont nourris avec les mêmes produits contaminés avec ces pesticides.

Les résultats ont montré une prédominance du DDT et de l'Endosulfane. Les concentrations relativement élevées du DDT sont probablement en relation avec les utilisations récentes. En effet, une fois absorbés chez les mammifères, le DDT et ses métabolites sont rapidement distribués via le sang à tous les tissus de l'organisme pour y être conservés et subissent un métabolisme partiel. A cause de leur liposolubilité, ces composés vont surtout s'accumuler dans les graisses. Le 4,4'- DDT subit une déchlorination réductive en $4,4^{\prime}$ - DDD, qui est ensuite dégradé en DDA (acide chlorophényl acétique) puis excrété dans les urines (Kitamura et al., 2002). Il peut être converti en 4,4'- DDE par déshydrochlorination mais à une vitesse très lente. Car ce métabolite est très retenu dans les tissus adipeux (Tebourbi et al., 2006), ce qui en partie peut justifier la prédominance du DDT dans les échantillons de lait de cette étude. Gbaguidi et al., (2011) expliquent le recours abusif, des agriculteurs aux pesticides organochlorés comme l'endosulfane et même le DDT au Bénin par rapport à la difficulté du choix d'un insecticide en lutte anti-vectorielle. Asssogba (2014) signalait aussi dans ses travaux, que face aux difficultés liées à la mise en place tardive des insecticides, à la pénibilité de la lutte étagée ciblée, les exploitants sont contraints à l'usage de pesticides en surdose ou au recours aux pesticides prohibés tels que l'endosulfane. Akpo et al., (2016) expliquent son utilisation systématique et intense pour lutter contre Hélicoverpa armigera qui est un ravageur du cotonnier. Le même constat a été fait par Agbohessi et al., (2012), que l'endosulfane a été réintroduit pour lutter contre cet ennemi redoutable du cotonnier à partir de 2000 au Bénin. Ledit ravageur sévit dans les plantations de cotonniers de la zone d'étude dont une partie est frontalière avec la Côte d'Ivoire et explique son utilisation par les producteurs à cause de leur connaissance de son efficacité contre ce dernier. En dépit de leur interdiction ces pesticides continuent d'être utilisés par les producteurs maliens. Cet usage frauduleux résulte de leur faible coût d'achat comparativement à celui des pesticides autorisés et homologués plus chers à l'achat.

Les auteurs Traore et al. (2008), avaient signalé dans leurs travaux, que la quantification des résidus des pesticides dans les échantillons de lait était la conséquence évidente de l'utilisation des matières actives comme l'endosulfane, le DDT, la dieldrine et le lindane en production agricole. La mise en évidence de la présence des résidus des pesticides organochlorés dans les échantillons de lait peut de ce fait s'expliquer par la contamination des sols, pâturages, des eaux souterraines et de surface dans l'environnement immédiat des champs de coton (Najim et al., 2003) ainsi que du traitement des animaux laitiers contre les parasites internes et externes (David, 2004). L'étude de Kouadio et al., (2014) a présenté des teneurs résiduelles moyennes de dieldrine, et d'endosulfane et du DDT dans le lait caillé 
en Côte d'Ivoire. Ces résultats ont été relativement supérieurs pour le DDT et nettement inférieurs pour l'endosulfane et la dieldrine. Cette différence peut être due aux spéculations de rente cultivées (le cacao, café et le coton) en Côte d'Ivoire contre le coton au Mali qui reçoivent toutes des traitements avec les pesticides mais à des doses et fréquences différentes. Une autre raison peut être, au Mali, l'alimentation du bétail est beaucoup axée sur les sous-produits agricoles (tiges, tourteaux et graines de coton, son et tiges des céréales, etc.) comme aliments grossiers des vaches laitières provenant des parcelles traitées avec des pesticides. De plus les graines de coton, après extraction de leur huile sont transformées en tourteaux utilisé comme aliment concentré et distribué durant toute l'année aux animaux. L'usage des pesticides dans la production agricole et le traitement vétérinaire ainsi que l'alimentation des animaux avec des aliments contenant des résidus de pesticides contribuent à contaminer directement les animaux qui vont produire un lait renfermant des résidus de pesticides.

\section{Conclusion}

Les résultats des analyses effectuées en deux saisons de prélèvement sur 100 échantillons de lait de vache provenant de deux grandes zones productrices de coton et de lait des régions de Koulikoro et Sikasso, ont révélé, dans la majorité des cas, une contamination par des résidus de 5 pesticides organochlorés. Ils ont aussi montré que les échantillons de lait provenant de la zone de forte production cotonnière (Koumantou, Ouéléssebougou et Sido) étaient plus contaminés que ceux de la zone de faible production cotonnière (Kasséla et Tienfala). Cependant, aucune influence de cette contamination par la saison n'a été déterminée. Il ressort de ces résultats que les pesticides les plus rencontrés sont le DDT et l'Endosulfane alors que le lindane a été très peu quantifié. Ils mettent en évidence la présence des résidus de ces pesticides dans la chaîne alimentaire à travers le lait de vache bien que leur utilisation est interdite au Mali depuis des décennies. Ils posent la nécessité des contrôles analytiques systématiques et la comparaison de ces derniers avec les doses limites tolérables pour les résidus de pesticides dans le lait.

\section{CONFLIT D'INTERETS}

Il n'existe aucun conflit d'intérêt entre les auteurs de ce manuscrit.

\section{CONTRIBUTIONS DES AUTEURS}

$\mathrm{BM}$ et $\mathrm{AB}$ ont assuré à la fois la rédaction de la méthodologie et la coordination scientifique de ce travail. $\mathrm{OH}$, FD et IT ont contribué de façon équitable au traitement technique des échantillons et la supervision des travaux d'analyse. IK, SK et AS ont réalisé la cartographie de la zone d'étude et l'analyse statistique des résultats des analyses. Tous les auteurs ont participé à la rédaction du manuscrit et ont apporté leur correction.

\section{REMERCIEMENTS}

Nos remerciements vont à l'endroit de l'ANSA, des responsables des minilaiteries, chefs de zone et agents d'encadrement des services techniques (CMDT, OHVN et Productions animales), producteurs et revendeurs de lait des 5 sites (Kasséla, Koumantou, Ouéléssebougou, Sido et Tienfala) pour leur contribution dans la réalisation des travaux du terrain.

\section{REFERENCES}

Adjagodo A, Tchibozo MAD, Kelome NC, Lawani R. 2016. Flux des polluants liés aux activités anthropiques, risques sur les ressources en eua de surface et la chaine trophique à travers le monde: synthèse bibliographique. Int. J. Biol. Chem. Sci., 10(3): 1459-1472. DOI: http://dx.doi.org/10.4314/ijbcs.v10i3.43.

Agbohessi PT, Toko II, Attakpa EY, Kestemont P. 2012. Synthèse des 
caractéristiques physico-chimiques et effets écotoxicologiques du Tihan 175 O-TEQ utilisé dans la protection phytosanitaire du cotonnier au Bénin. Int. J. Biol. Chem. Sci., 6(5):2280-2292. DOI: http://dx.doi.org/10.4314/ijbcs.v6i5.34.

Akpo SK, Coulibaly LS, Coulibaly L, Savane I. 2016. Evolution temporelle de l'utilisation des pesticides en agriculture tropicale dans le bassin versant de Marahoué, en Côte d'Ivoire. International Journal of Innovation and Applied Studies, 14(1): 121-131.

Amadou H, Hülsebusch C, Berthé A, Schlecht E. 2014. Safety of horticultural and livestock products in two medium-sized cities of Mali and Burkina Faso. African Journal of Agricultural Research, 9(8):735-745. DOI: 10.5897/AJAR2013.6897.

Assogba SCG. 2014. Représentations de l'environnement et adoption des pratiques durables de production par les cotonculteurs du Bénin. Thèse de Doctorat, Université de Liège/Gembloux Agro-Bio Tech, Belgique, 204p.

Bonfoh B, Dem S, Keita O, Delorenzi S, Traore H, Simbe CF, Alfaroukh IO, Farah Z, Nicolet J, Zinsstag J. 2003. Assessment of antibiotic residues by microbial inhibitor tests in fresh cow milk sold in Bamako (Mali). Milchwissenschaft, 58 (5-6): 304-307.

CMDT (Compagnie Malienne de Développement des Textiles). 2018. Rapport d'activités annuel 2017. 182p.

David HW. 2004. Pesticide, Veterinary and Other Residues in Food. Woodhead Publishing Limited and CRC Press LLC, Cambridge England.

Dem SB, Cobb J, Mullins DE. 2007. Pesticide Residues in Soil and Water from Four Cotton Growing Areas of Mali, West Africa. J. Agric., Food Environ. Sci., 1(1): 1-12.
DNPIA (Direction Nationale des Productions et Industries Animales du Mali). 2012. Document du projet de stratégie de valorisation du lait cru local au Mali. $44 \mathrm{p}$.

DNPIA (Direction Nationale des Productions et Industries Animales du Mali). 2016. Rapport annuel d'activités 2015. 114p.

Gbaguidi MAN, Soclo HH, Issa YM, Fayomi B, Dognon R, Agagbe A, Bonou C, Youssao A, Dovonou LF, Sanni A. 2011. Evaluation quantitative des résidus de pyréthrinoïdes, d'aminophosphate et de triazines en zone de production de coton au Bénin par la méthode ELISA en phase liquide : cas de la rivière Agbado. Int. $J$. Biol. Chem. Sci., 5(4):1476-1490. DOI: http://dx.doi.org/10.4314/ijbcs.v5i4.14.

Grillet N, Grimaud P, Loiseau G, Wesuta M, Faye B. 2005. Qualité sanitaire du lait cru tout au long de la filière dans le district de Mbarara et la ville de Kampala en Ouganda. Revue Elev. Méd. Vét. Pays trop., 28(4): 245-255.

Kitamura S, Shimizu Y, Shiraga Y, Yoshida M, Sugihara K, Ohta S. 2002. Reductive Metabolism of p,p'- DDT and o,p'- DDT by Rat Liver Cytochrome P450. Drug Metabolism and Disposition, 30(2): 113$118 . \quad$ DOI: https: //doi.org/10.1124/dmd.30.2.113.

Kouadio DL, Ehouman SGA, Soro BD, Diarra M, Doumbia ML, Meite L, Koné M, Dembélé A, Traore SK. 2014. Contamination du lait caillé et de l'œuf consommé en Côte d'Ivoire par des pesticides organochlorés. Afrique SCIENCE, 10(4) : 61-69.

ISO 3890-1. 2009. Détermination des résidus de composes organochlorés (pesticides)Partie 1: considérations générales et méthodes d'extraction, IDF-75-1.

ISO 3890-2. 2009. Détermination des résidus de composes organochlorés (pesticides)Partie 2: méthodes d'essais pour la purification des extraits bruts et tests de confirmation, IDF-75-2. 
Mensah SEP, Koudandé OD, Sanders P, Laurentie M, Mensah GA, Abiola FA. 2014. Résidus d'antibiotiques et denrées d'origine animale en Afrique : risques de santé publique. Rev. Sci. Tech. Off. Int. Epiz., 33(3): 975-986.

Najim A, Hofman E, Gnamou KM. 2003. Les entreprises face aux enjeux du développement durable. Kharthala, Paris.

PASP. Programme Africain relatif aux Stocks de Pesticides obsolètes. 2009. Rapport annuel, $47 \mathrm{p}$.

Savadogo PW, Traore O, Topan M, Tapsoba KH, Sedogo PM, Bonzi-Coulibaly YL. 2006. Variation de la teneur en résidus de pesticides dans les sols de la zone cotonnière du Burkina Faso. J. Afri. Sci. l'Environ., 1 : 29-39.

Tchamadeu NN, Nkontcheu DBN, Nana ED. 2017. Evaluation des facteurs de risques environnementaux liés à la mauvaise utilisation des pesticides par les maraîchers au Cameroun: le cas de
Balessing à l'Ouest Cameroun. Afrique SCIENCE, 13(1) : 91-100.

Tebourbi O, Driss M R, Sakly M, Rhouma K B. 2006. Metabolism of DDT in different Tissues of Young Rats. Journal of Environmental Science and Health, 41(2): 167-176.

TECA-FAO. 2016. La gestion des ravageurs et des maladies en agriculture biologique.

www.teca.fao.org/fr/read/8575, consulté le 15 novembre 2017, 12:30.

Traore SK, Dembélé A, Koné M, Mambo V, Lafrance P, Bekro YA, Houenou P. 2008. Contrôle des pesticides organochlorés dans le lait et produits laitiers: Bioaccumulation et risque d'exposition. Afrique SCIENCE, 04(1): 87-98.

UNEP/Chemicals Branch DTIE/POP. 2012. Supporting the implementation of the Global Monitoring Plan in the West African Region. Final Report, 40p. 